it turned out to be a typical hour-glass contraction of the stomach. The diagnosis chiefly depended on the absence of the marked dilatation of the stomach which would occur in py loric constriction and in the bubbling of the fluid through a narrow orifice which was heard, not in the situation of the pylorus, but about the centre of the stomach. The further point of interest is in the treatment. One was very much tempted to excise the contracted portion of the stomach altogether and to stitch the walls together on each side and no doubt that would have been followed by complete recovery without any risk of recurrence of the contraction, for of course it must be admitted that there may possibly be an increased contraction still and some recurrence of the symptoms, but the patient was in an extremely feeble condition and it did not seem justifiable to subject her to the prolonged operation which would have been involved in this procedure, nor was I quite sure that the circulation in the stomach after complete bisection would have been quite satisfactory. On the other hand, the operation performed answered all expectations and the fact that the symptoms have now been absent for several months and that she is able to take solid food without any trouble leads me to hope that the result will be a permanent success.

\section{KENSINGTON INFIRMARY.}

A CASE OF GASTROTOMY FOR REMOVAL OF A FOREIGN BODY.

(Under the care of Dr. H. Percy Potter.)

THE necessity for gastrotomy for the removal of a foreign body from the stomach can only arise in cases in which the body is nnable or unlikely to pass through the pylorus. Probably the most frequent foreign body is a coin and in the majority of instances it has no difficulty in leaving the stomach; but in the case of a long thin body the chance of getting through the pylorus is very small and the object is either retained indefinitely in the stomach or finds its way, after causing ulceration, through the walls of the stomach, as in a case we have recorded lately which was under the care of Mr. Morgan at Charing-cross Hospital. ${ }^{1}$ In the case of such a body it is very necessary that an operation should be performed as early as possible. In all these cases, however, the evidence of the presence of the foreign body is not very satisfactory and until the introduction of the Roentgen rays no method existed by which the surgeon could assure himself that a foreign body was really present, so that he often hesitated to operate when an operation was very desirable.

A well-nourished woman, aged thirty years, was admitted to the Kensington Infirmary on Jan. 13th stating that she had intentionally forced a button-hook about four inches long down her throat. She was quiet and reserved and gave every detail of the act in a rational manner, so her statement was believed. The hook could neither be seen nor felt. A bougie was without difficulty passed into the stomach. She was kept under observation till 8 P.M. and then taken to Dr. Low, who produced by the Roentgen rays three unmistakeable negatives of the foreign body in situ. It lay in the epigastrium on the left of the middle line, the hook portion pointing downwards and inwards about an inch outside the first lumbar vertebra. On the morning of the 14th a median vertical incision three and a half inches long was made between the ziphoid cartilage and umbilicus, opening the peritoneal cavity. Upon introducing two fingers to the left the loop of the button-hook was found deeply situated; the hook portion was manipulated towards the plane of the abdominal incision, steadied between the thumb and finger, cut down upon, and removed. ${ }^{2}$ The mucous membrane was united by a continuous silk suture, the mucous and serons coats by Lembert's suture, and the parietal incision by interrupted silk and silkworm-gat stitches. The wound was then dressed with blue gauze steeped in perchloride and was covered with a many-tailed (or domett) bandage. The operation was practically bloodless and no sponges were introduced into the abdomen. An hour after the operation the patient romited blood-stained fluid; in the afternoon

1 The LanceT, Feb. 5th, 1898, p. 355

2 The button-hook was of the cheap steel variety, shaped out of a niece of soft steel wire, and measured four and a half inches in length and one inch in breadth at the loop. during the ntervals of sleep, she complained of pain which was relieved by an injection of morphia. A nutrient enema of peptonised milk was given in the evening and repeated every four hours. Ten drops of laudanum in hot water were given by the mouth at 9 P M. On the 16 th the wound was dresse $d$; the epigastrium was slightly distended ; there was no pain, A lemon was sucked to allay thirst. Two teaspoonfuls of peptonised milk were taken every hour; this quantity was gradually increased till the 24th when the

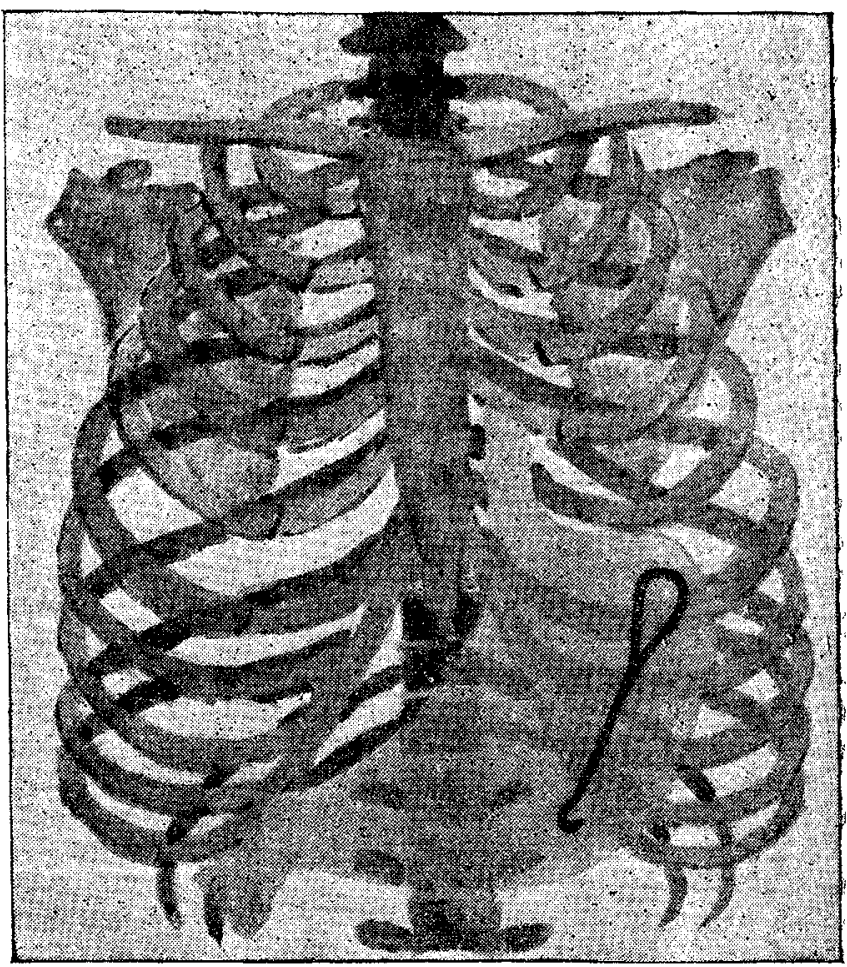

patient had an egg with bread and butter and enjoyed some "beef-cacao." On the 25th the sutures were remored and she was allowed fish on the 27 th. The wound was completely healed on the 29th and the patient got up on Feb. 4th convalescent. There was absence of fever and complication of any kind throughout the case.

Remarks by Dr. POTTER.-The operation of gastrotomy has frequently been performed for the removal of a foreign body from the stomach and provided these cases are taken in hand early the results are favourable. It must be difficult in most cases to determine with accuracy by external manipulation the site or even the presence of a foreign body which has been swallowed, but by the use of the Roentgen rays there is a new light thrown upon these cases and a decisive verdict given in respect of the necessity for an operation. The difficuliy of obtaining good photographic results consists in the thickness of the abdominal walls and contents and the movements of respiration and likewise of the stomach itself. The accompanying illustration is reproduced from a drawing of the skiagram, which is somewhat indistinct and blurred but sufficiently definite to determine the presence of something which ought not to be there. My thanks are due to Mr. Osborn and Mr. Lloyd for their valuable assistance.

The Prince of Wales's Hospital Fund for LoNDON.-We have received a copy of the "Subscription Book and Stamp Album," issued in connexion with, and in support of, the Fund, together with an antograph letter from H.R.H. the Princess of Wales in which she makes a hearttouching appeal to the children of the United Kingdom to assist the Fund by contributing their donations howeret small.

Cornwarl Country Asylum.-The annugl report of the Cornwall County Asylum at Bodmin showed that during 1897 the average number of patients had been 770, the highest yet recorded. The temporary accommodation provided near the asylum for 18 male patients is occupied and there are 22 patients boarded out at the Plymouth Borough Asylum. The report adds that the isolation hospital is to be commenced immediately. The committee refer in terms of regret to the resignation of the medical superintendent, Mr. Richard Adams, M.R.C.S. Eng. L.R.C.P. Edin., after forty years of valued servics. 